RU-NHETC-2012-12

UTTG-09-12

\title{
Jet Extinction from Non-Perturbative Quantum Gravity Effects
}

\author{
Can Kilic ${ }^{1}$, Amitabh Lath $^{2}$, Keith Rose ${ }^{2}$, Scott Thomas ${ }^{2}$ \\ ${ }^{1}$ Theory Group, Department of Physics and Texas Cosmology Center \\ The University of Texas at Austin \\ Austin, TX 78712 \\ ${ }^{2}$ Department of Physics \\ Rutgers University \\ Piscataway, NJ 08854
}

\begin{abstract}
The infrared-ultraviolet properties of quantum gravity suggest on very general grounds that hard short distance scattering processes are highly suppressed for center of mass scattering energies beyond the fundamental Planck scale. If this scale is not too far above the electroweak scale, these non-perturbative quantum gravity effects could be manifest as an extinction of high transverse momentum jets at the LHC. To model these effects we implement an Extinction Monte Carlo modification of the Pythia event generator based on a Veneziano form factor with a large absorptive branch cut modification of hard QCD scattering processes. Using this we illustrate the leading effects of extinction on the inclusive jet transverse momentum spectrum at the LHC. We estimate that an extinction mass scale of up to roughly half the center of mass beam collision energy could be probed with high statistics data. Experimental searches at the LHC for jet extinction would be complementary to ongoing searches for the related phenomenon of excess production of high multiplicity final states.
\end{abstract}




\section{Introduction}

The scattering of high energy particles in theories of quantum gravity differs in qualitatively significant ways from scattering in local quantum field theories [1, 2]. The holographic infrared-ultraviolet properties of quantum gravity imply that at center of mass energies beyond the fundamental Planck scale, scattering is dominated by non-perturbative processes that result in final states with a high multiplicity of low energy particles. In the very high energy limit, this universal feature of quantum gravity scattering can be understood in terms of semi-classical formation of massive black hole intermediate states that subsequently undergo low energy Hawking evaporation [1]. Concomitant with the predominance of high entropy non-perturbative states at high energies is an exponential suppression of perturbative hard scattering processes that give low multiplicity final states [1, 3]. The essential extinction of hard short distance scattering processes in this regime implies that for incident colliding particles of any center of mass energy, the energies of individual final state particles are effectively bounded by the fundamental Planck scale - a striking marker of the infrared-ultraviolet nature of quantum gravity.

If the fundamental Planck scale is not too far above the electroweak scale [4, 5, 6] certain features of quantum gravity could in principle be subject to experimental investigation at high energy colliders. Most proposals for probing non-perturbative quantum gravity effects in high energy collisions have focussed on production of high entropy intermediate states, colloquially referred to as microscopic black holes, that decay to high multiplicity final states [3, 7, 8]. At the Large Hadron Collider (LHC) the CMS and ATLAS collaborations have conducted searches for excess high multiplicity final states [9, 10, 11, 12]. Other proposals have involved long distance scattering in perturbative quantum gravity [13, 14] and perturbative string theory effects [15, 16]. The CMS and ATLAS collaborations have conducted searches for perturbative string excitations and other purported quantum gravity resonance phenomena in the di-jet mass spectrum [17, 18, 19, 20].

In this paper we investigate the possibility of observing the extinction of hard scattering processes in high energy collisions from non-perturbative quantum gravity effects. At the LHC the leading hard scattering process at high energies is jet production from QCD interactions. Extinction from non-perturbative quantum gravity effects should therefore be most apparent as a suppression of large invariant mass scattering processes involving jets, or equivalently a suppression of large transverse momentum in the inclusive jet spectrum. Such features should be a rather direct consequence of the infrared-ultraviolet properties of non-perturbative quantum gravity [1, 3]. Searches for the extinction of high energy hard 
scattering processes would be complementary to searches for the appearance of high multiplicity high energy processes. Both features would be associated with scattering energies far beyond the fundamental Planck scale. Other features associated with physics near the fundamental scale, such as resonance phenomena, depend on model dependent aspects of the full theory of quantum gravity. As a practical matter, current or planned high energy colliders are unlikely to provide energies well in excess of the fundamental Planck scale. So it is worth exploring different signatures for the onset of non-perturbative quantum gravity effects, since details of which might provide the most sensitive probe at energies of order the fundamental scale are likely to depend on specific attributes of the full theory of quantum gravity.

The specific model we utilize to quantify the extinction of hard scattering is a Veneziano form factor modification of QCD processes with a large absorptive damping component coming from a branch cut in the scattering amplitudes. This model can be motivated from some underlying features of the Veneziano amplitude [21]. The scattering of strings described by the Veneziano amplitude is dominated at energies well above the string scale by high entropy intermediate states [22, 23]. For energies above the string scale, but below the Planck scale, these states correspond to excited perturbative strings, with widths, or equivalently damping, that grow with energy. Above the Planck scale the excited states become strongly self coupled, with large widths and damping. In this regime the Veneziano amplitude effectively represents scattering into a continuum of high entropy intermediate states that have suppressed overlap with low multiplicity final states. Imposing a large absorptive branch cut in the Veneziano amplitude essentially eliminates the perturbative regime, and yields a model for the extinction of low multiplicity scattering processes at energies above a fundamental scale. This model is not meant to provide a complete description of non-perturbative quantum gravity processes in specific theories of quantum gravity, but only to provide a representation of the onset of extinction in low multiplicity scattering processes.

One nice feature of the large damping Veneziano form factor model employed here is that it smoothly matches onto QCD as the fundamental scale is taken large. This is in contrast to models with black hole geometric cross sections [3, 7, 8] that have been used to parameterize the results of LHC searches for high multiplicity final states [9, 10, 11, 12]. These models are well founded by general considerations of semi-classical Einstein gravity for center of mass scattering energies asymptotically beyond the fundamental scale. But the models do not match smoothly onto lower energy scattering, and their direct application to energies just above the fundamental scale may be dubious. In this sense quantifying the 
results of extinction searches in terms of a fundamental scale with the model utilized here could be considered to be more conservative than those from high multiplicity searches with the fundamental scale described in terms of black hole geometric cross sections. It should also be noted that the quantitative results from experimental searches for various types of new physics associated with a fundamental scale, such as high multiplicity final states, resonance phenomena, and the extinction effects presented here, can not generally be compared directly since the models used to quantify these disparate processes make different exclusive assumptions about what effects are important near the fundamental scale.

The Veneziano form factor model for extinction of leading order (LO) QCD scattering processes is detailed in the next section. In section 3 we describe an Extinction Monte Carlo modification of the Pythia event generator based on the Veneziano form factor model. We use this Monte Carlo to simulate the leading effects of extinction on the inclusive jet transverse momentum spectrum at the LHC, and to roughly estimate the statistical sensitivity of $7 \mathrm{TeV}$ and future $13 \mathrm{TeV}$ measurements to the extinction mass scale. We also suggest a simple and conservative phenomenological form factor modification of next to leading order (NLO) QCD predictions of the inclusive jet transverse momentum spectrum that could be used to quantify the results of searches for extinction.

\section{Modeling Extinction}

Simulation of jet extinction requires a phenomenological model for the modification of low multiplicity scattering processes. In the next subsection, form factor modifications for all quark and gluon $2 \rightarrow 2$ scattering processes of QCD based on a modified form of the Veneziano amplitude with large absorptive branch cut are introduced. These form factors provide a simple model representation of the general properties expected from the existence of strongly self coupled high entropy intermediate states. In the following subsection the salient quantitative features of the extinction form factor model are presented.

\subsection{Veneziano Form Factor Model}

The $2 \rightarrow 2$ scattering amplitude for any QCD process involving massless particles $p_{1}, p_{2}, p_{3}, p_{4}$ may be represented as a color ordered helicity amplitude $\mathcal{A}\left(p_{1}, p_{2}, p_{3}, p_{4}\right)_{\mathrm{QCD}}$. The extinction model employed here is defined through these scattering amplitudes modified by a 
universal form factor in adjacent color ordered kinematic channels

$$
\mathcal{A}\left(p_{1}, p_{2}, p_{3}, p_{4}\right)=\mathcal{A}\left(p_{1}, p_{2}, p_{3}, p_{4}\right)_{\mathrm{QCD}} \tilde{V}\left(x_{12}, x_{23}\right)
$$

where $x_{A B}=\left(p_{A}+p_{B}\right)^{2}=2 p_{A} \cdot p_{B}$ with all momenta incoming. The purpose here is not to derive a complete expression for the form factor directly from some underlying theory of quantum gravity, but rather to develop a model expression based on general principles that captures the salient features of the onset of extinction.

In order to provide a useful model for the onset of extinction of $2 \rightarrow 2$ scattering processes, the form factor $\widetilde{V}\left(x_{12}, x_{34}\right)$ should satisfy certain general properties. The first is that the extinction effects should be characterized by an extinction mass scale $M_{E}$. And for the scattering amplitudes (1) to reduce to those of QCD at low energies, the form factor should approach unity for small values of the invariant momenta

$$
\lim _{x_{12} \text { or } x_{34} \ll M_{E}} \tilde{V}\left(x_{12}, x_{34}\right)=1
$$

The form factor may then be written in terms of a dimensionless function

$$
\widetilde{V}\left(x_{12}, x_{34}\right)=V\left(x_{12} / M_{E}^{2}, x_{34} / M_{E}^{2}\right)
$$

In order to represent the effects of extinction we also require that the form factor be bounded from above by unity

$$
V\left(x_{12}, x_{34}\right) \leq 1
$$

with significant deviations from unity only for $x_{12}, x_{34} \gtrsim 1$. A third requirement that we impose is crossing symmetry

$$
V\left(x_{12}, x_{34}\right)=V\left(x_{34}, x_{12}\right)
$$

This ensures that extinction effects appear in all kinematic and color channels. As a simple ansatz satisfying the crossing symmetry property (5) we consider form factors that factorize into a product of identical functions of the kinematic invariant in each channel, times an overall normalization that is a function of the sum of the kinematic invariants. The requirement (2) of approach to unity for small values of the kinematic invariants then determines the form factor in terms of a single function

$$
V\left(x_{12}, x_{34}\right)=\frac{V\left(x_{12}\right) V\left(x_{34}\right)}{V\left(x_{12}+x_{34}\right)}
$$




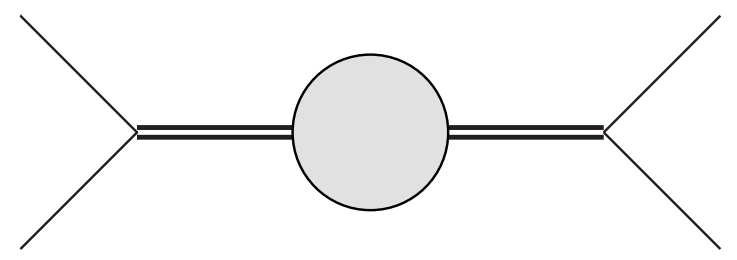

Figure 1: Representation of the modification of QCD $2 \rightarrow 2$ scattering processes from the effects of heavy states that couple to intermediate multi-particle states. The single external lines represent quark and gluon scattering states. The double lines represent heavy unstable states in the given kinematic and color channel. The grey blob represents high entropy intermediate states that produce a large absorptive branch cut in the imaginary part of the amplitude and lead to an extinction of the $2 \rightarrow 2$ scattering probability at high energies.

Another general requirement is dictated by the form of analytic continuation of the scattering amplitudes (1) for complex values of invariant momenta. The real parts of the QCD amplitudes are continuous when the kinematic invariants are extended into the complex plane. Preserving this property for the modified amplitudes (1) requires that the form factor satisfies Hermitian analyticity

$$
V\left(z_{12}^{*}, z_{34}^{*}\right)=\left[V\left(z_{12}, z_{34}\right)\right]^{*}
$$

This property introduces an absorptive branch cut in the imaginary part of the form factor, and is a crucial feature in modeling extinction of $2 \rightarrow 2$ scattering processes coming from the effects of high entropy intermediate states. Other requirements are provided by dispersion relations between the real and imaginary parts of the form factor. These integral relations constrain the behavior of the form factor for asymptotic values of the kinematic invariants. Since the purpose here is only to provide a phenomenological model for the onset of extinction that is applicable to kinematic invariants of order the extinction scale, $M_{E}$, we do not consider dispersion relation restrictions on the form factor that would apply outside this kinematic regime.

Local quantum field theory is not ameanable to a complete description of high energy scattering processes in quantum gravity. However, all the ingredients necessary for a phenomenological form factor model for the onset of extinction that satisfy the requirements listed above are available in local quantum field theory. So we employ this language to illustrate the elements of a model. We begin by introducing operators $\mathcal{O}_{a}$ that create and annihilate heavy unstable states with the same kinematic and color quantum numbers that appear in all channels of QCD $2 \rightarrow 2$ scattering processes. These operators couple to com- 
posite operators $J_{a i}$ that create and annihilate multi-particle states through interactions

$$
\sum_{a, i} g_{a i} \int d^{4} x J_{a i} \mathcal{O}_{a}+\text { h.c. }
$$

Among the operators $J_{a i}$ are ones made out of quark and gluon fields that allow the heavy states to be exchanged in every kinematic and color channel in $2 \rightarrow 2$ quark and gluon scattering processes. A schematic representation of the modification of QCD $2 \rightarrow 2$ scattering processes from the intermediate multi-particle states through the exchange of the heavy states is shown in Fig. 1 .

The effects of the intermediate multi-particle states in a given channel are contained within the normalized one-particle-irreducible two-point function for the composite operators that appear in that channel

$$
i \sum_{a, i} g_{a i}^{2} \int d^{4} x e^{i p \cdot x}\left\langle 0\left|T\left\{J_{a i}(x) J_{a i}^{\dagger}(0)\right\}\right| 0\right\rangle_{1 \mathrm{PI}} \equiv\left\{\begin{array}{cl}
-M^{2}\left(p^{2}\right) & \text { Boson } \\
-M\left(p^{2}\right) & \text { Fermion }
\end{array}\right.
$$

In the space-like region $p^{2}<0$ and in the time-like region below threshold for production of intermediate states $p^{2}<p_{0}^{2} \geq 0$ there are no contributions from on-shell intermediate states, and the two-point function (9) is strictly real with no absorptive imaginary component. For the analytic continuation of the two-point function into the complex plane this implies that for $\operatorname{Re}(z)=p^{2}<p_{0}^{2}$ along the real axis in this region

$$
M^{2}(z)=\left[M^{2}\left(z^{*}\right)\right]^{*}
$$

Extending the relation (10) to the entire complex plane implies that the imaginary component of the two-point function has a branch cut in the time-like region for real $x=p^{2} \geq p_{0}^{2}$ on the real axis above threshold of

$$
\operatorname{Disc} M^{2}(x) \equiv M^{2}(x+i \epsilon)-M^{2}(x-i \epsilon)=2 i \operatorname{Im} M^{2}(x+i \epsilon)
$$

The discontinuity is proportional to the total production rate $\Gamma\left(p^{2}\right)$ of on-shell intermediate multi-particle states from decay of the massive states

$$
\sqrt{\left|p^{2}\right|} \Gamma\left(p^{2}\right)=\frac{i}{2} \operatorname{Disc} M^{2}\left(p^{2}\right)=-\operatorname{Im} M^{2}\left(p^{2}+i \epsilon\right)
$$

Unitarity requires that $i$ Disc $M^{2}\left(p^{2}\right) \geq 0$ along the branch cut. The decay rate 12 represents a transfer of scattering probability from particular exclusive two-body final states to inclusive multi-particle final states. This feature allows for a representation of the effects 
of high entropy intermediate states in the phenomenological model through the absorptive branch cut 11].

The complete modification of QCD $2 \rightarrow 2$ scattering processes in a given kinematic channel from exchange of the heavy states with couplings (8) to multi-particle intermediate states are obtained by summing the geometric series of all one-particle-reducible diagrams for the propagation of the heavy states in that channel connected by intermediate oneparticle-irreducible multi-particle contributions to the two-point function (9). The $2 \rightarrow$ 2 scattering diagram with two propagators for the heavy states connected by a single insertion of the two-point function (9) is illustrated in Fig. 11. The form factor function in a given kinematic channel arising from the geometric sum may be represented as a spectral decomposition over the heavy states

$$
V(z)=1+\int d m^{2} \frac{\rho\left(m^{2}\right)}{z-m^{2}-M^{2}(z)}
$$

where $\rho\left(m^{2}\right)$ is the spectral density of the heavy intermediate states with mass $m$. The real part of the two-point function (9) from the intermediate multi-particle states may be absorbed into the definition of the heavy state masses, $m^{\prime 2}=m^{2}-\operatorname{Re}\left(M^{2}(z)\right)$, so that the form factor has functional dependence $V(z)=f\left[z-i \operatorname{Im}\left(M^{2}(z)\right)\right]$. Motivated by the general properties of the Venziano amplitude outlined in the introduction, we choose a gamma function with massless intercept as our model function to represent the effects of heavy intermediate states, $f(y)=\Gamma(1-y)$. With this model assumption, our dimensionless form factor (6) is then

$$
V\left(z_{12}, z_{34}\right)=\frac{\Gamma\left[1-z_{12}+i \operatorname{Im}\left(M^{2}\left(z_{12}\right)\right)\right] \Gamma\left[1-z_{34}+i \operatorname{Im}\left(M^{2}\left(z_{34}\right)\right)\right]}{\Gamma\left[1-z_{12}-z_{34}+i \operatorname{Im}\left(M^{2}\left(z_{12}+z_{34}\right)\right)\right]}
$$

The branch cut (11) in the two-point function (9) ensures that the form factor (14) satisfies the Hermitian analyticity property (7) for complex values of the kinematic invariants. For real values of the momenta, proper ingoing and outgoing boundary conditions are obtained from the form factor with kinematic invariants shifted just above the real axis, $V\left(x_{12}, x_{34}\right) \equiv V\left(x_{12}+i \epsilon, x_{34}+i \epsilon\right)$ with $\epsilon \rightarrow 0$. For $\operatorname{Im}\left(M^{2}(z)\right)=0$ the form factor 14 reduces to the Veneziano amplitude [21] with massless intercept.

The final ingredient needed to specify the full functional dependence of the form factor is a model for the absorptive imaginary component of the two-point function (9) coming from intermediate on-shell multi-particle states. This absorptive component represents the transfer of scattering probability to inclusive multi-particle final states and is crucial 
in modeling the effects of extinction. Consistent with the analyticity condition (10) and multi-particle threshold at $x_{0}=p_{0}^{2}=0$ we parameterize the branch cut (11) along the positive real axis as

$$
-\frac{i}{2} \operatorname{Disc} M^{2}(x)=\operatorname{Im} M^{2}(x+i \epsilon)=\alpha x^{n} \theta(x)
$$

where $\theta(y)$ is the Heaviside step function. Following previous work [8] we adopt a phenomenological value of $n=1$ for the exponent of the momentum dependence of the branch cut. Although this particular value for the exponent does not correspond to an analytic two-point function in the entire complex plane without additional unphysical branch cuts away from the real axis, it does provide a simple model parameterization for the magnitude of the absorptive phase in the physically important regime for extinction effects corresponding to kinematic invariants of order the extinction scale. With the parameterization (15) with $n=1$, the decay rate 12 of the massive states to on-shell multi-particle states is $\Gamma\left(m^{2}\right)=\alpha m$. The damping parameter $\alpha$ therefore represents the dimensionless width of the heavy states. The extinction property (4) is obtained for a constant $\alpha=1$. This value is used in the results and simulations presented below.

In the following subsections the squared amplitudes for all QCD $2 \rightarrow 2$ scattering processes organized by different choices of the external particles with the form factor modification prescription (1) are presented. Similar results for the specific case of Veneziano form factor modifications without branch cuts may be obtained from leading order perturbative open-string scattering disk amplitudes [16] but with some model dependence for amplitudes that involve only quarks and/or anti-quarks.

\subsubsection{Two Quarks of One Flavor and Two Quarks of Another Flavor}

The unique color ordered helicity amplitudes with two quarks of one flavor and two quarks of another flavor may be written

$$
\mathcal{A}\left(\bar{q}_{1}^{-i}, q_{2}^{+j}, \bar{q}_{3}^{\prime \pm k}, q_{4}^{\prime \mp \ell}\right)=A\left(\bar{q}_{1}^{-i}, q_{2}^{+j}, \bar{q}_{3}^{\prime \pm k}, q_{4}^{\prime \mp \ell}\right)\left(T^{a}\right)_{j}{ }^{i}\left(T^{a}\right)_{\ell}{ }^{k}
$$

with others related by charge conjugation or parity, where throughout helicity and momentum indicated in helicity amplitudes are all incoming. The $i, j, \ldots$ are (anti)fundamental indices, $a, b, \ldots$ are adjoint indices, and $T^{a}$ are fundamental generators of $S U(3)_{C}$ with normalization $\operatorname{Tr}\left(T^{a} T^{b}\right)=\delta^{a b}$. The QCD helicity amplitudes with color factors stripped off, written in terms of twistor products are related by permutations to a single amplitude

$$
A\left(\bar{q}_{1}^{-i}, q_{2}^{+j}, \bar{q}_{3}^{\prime-k}, q_{4}^{\prime+\ell}\right)_{\mathrm{QCD}}=g^{2} \frac{[13]\langle 42\rangle}{\langle 12\rangle[21]}
$$




$$
A\left(\bar{q}_{1}^{-i}, q_{2}^{+j}, \bar{q}_{3}^{++k}, q_{4}^{\prime-\ell}\right)_{\mathrm{QCD}}=g^{2} \frac{[14]\langle 32\rangle}{\langle 12\rangle[21]}
$$

where the twistor products are defined in terms of contractions of the wave function spinors by $[A B] \equiv \chi^{\alpha}\left(p_{A}\right) \chi_{\alpha}\left(p_{B}\right)$ and $\langle A B\rangle \equiv \chi_{\dot{\alpha}}^{*}\left(p_{A}\right) \chi^{* \dot{\alpha}}\left(p_{B}\right)$ and satisfy $\langle A B\rangle=-\langle B A\rangle,\langle A B\rangle^{*}=$ $[B A]$. These color ordered helicity amplitudes may be averaged (summed) over initial (final) state color and helicity configurations to give the averaged squared matrix elements for $2 \rightarrow 2$ scattering processes involving two quarks of one flavor and two of another flavor with the form factor modification

$$
\begin{aligned}
& \left\langle\left|\mathcal{A}\left(q_{1} \bar{q}_{2} \rightarrow q_{3}^{\prime} \bar{q}_{4}^{\prime}\right)\right|^{2}\right\rangle=g^{4} \frac{4}{9} \frac{u^{2}+t^{2}}{s^{2}}|\widetilde{V}(s, t)|^{2} \\
& \left\langle\left|\mathcal{A}\left(q_{1} q_{2}^{\prime} \rightarrow q_{3} q_{4}^{\prime}\right)\right|^{2}\right\rangle=g^{4} \frac{4}{9} \frac{s^{2}+u^{2}}{t^{2}}|\widetilde{V}(t, u)|^{2} \\
& \left\langle\left|\mathcal{A}\left(q_{1} \bar{q}_{2}^{\prime} \rightarrow q_{3} \bar{q}_{4}^{\prime}\right)\right|^{2}\right\rangle=g^{4} \frac{4}{9} \frac{s^{2}+u^{2}}{t^{2}}|\widetilde{V}(s, t)|^{2} \\
& \left\langle\left|\mathcal{A}\left(\bar{q}_{1} \bar{q}_{2}^{\prime} \rightarrow \bar{q}_{3} \bar{q}_{4}^{\prime}\right)\right|^{2}\right\rangle=g^{4} \frac{4}{9} \frac{s^{2}+u^{2}}{t^{2}}|\widetilde{V}(t, u)|^{2}
\end{aligned}
$$

where $\langle A B\rangle[B A]=x_{A B}$, and $s=x_{12}=x_{34}, t=x_{13}=x_{24}$, and $u=x_{14}=x_{23}$ are the Mandelstam variables for the scattering order $12 \rightarrow 34$ indicated in the squared matrix elements. Each scattering matrix element squared 19,22 includes a sum over two independent helicity channels (not related by parity) of single helicity amplitudes squared in a single color channel.

\subsubsection{Four Quarks of the Same Flavor}

The color ordered helicity amplitudes with four quarks of the same flavor with either all the chiralities the same or two of one chirality and two of the other may be written respectively

$$
\begin{aligned}
& \mathcal{A}\left(\bar{q}_{1}^{-i}, q_{2}^{+j}, \bar{q}_{3}^{-k}, q_{4}^{+\ell}\right)=A\left(\bar{q}_{1}^{-i}, q_{2}^{+j}, \bar{q}_{3}^{-k}, q_{4}^{+\ell}\right)\left(T^{a}\right)_{j}{ }^{i}\left(T^{a}\right)_{\ell}{ }^{k} \\
& \mathcal{A}\left(\bar{q}_{1}^{-i}, q_{2}^{-j}, \bar{q}_{3}^{+k}, q_{4}^{+\ell}\right)=A\left(\bar{q}_{1}^{-i}, q_{2}^{-j}, \bar{q}_{3}^{+k}, q_{4}^{+\ell}\right)\left(T^{a}\right)_{j}{ }^{i}\left(T^{a}\right)_{\ell}{ }^{k}
\end{aligned}
$$

with others related by charge conjugation or parity. The QCD helicity amplitudes in this case with the color factor stripped off are identical to the amplitudes (17) and (18). These color ordered helicity amplitudes may be averaged (summed) over initial (final) state color and helicity configurations to give the averaged matrix elements squared for $2 \rightarrow 2$ scattering processes involving four quarks of a single flavor with the form factor modification

$$
\left\langle\left|\mathcal{A}\left(q_{1} \bar{q}_{2} \rightarrow q_{3} \bar{q}_{4}\right)\right|^{2}\right\rangle=g^{4} \frac{4}{9}\left[\frac{u^{2}+t^{2}}{s^{2}}+\frac{s^{2}+u^{2}}{t^{2}}-\frac{2}{3} \frac{u^{2}}{s t}\right]|\widetilde{V}(s, t)|^{2}
$$




$$
\begin{aligned}
& \left\langle\left|\mathcal{A}\left(q_{1} q_{2} \rightarrow q_{3} q_{4}\right)\right|^{2}\right\rangle=g^{4} \frac{4}{9}\left[\frac{s^{2}+u^{2}}{t^{2}}+\frac{s^{2}+t^{2}}{u^{2}}-\frac{2}{3} \frac{s^{2}}{t u}\right]|\widetilde{V}(t, u)|^{2} \\
& \left\langle\left|\mathcal{A}\left(\bar{q}_{1} \bar{q}_{2} \rightarrow \bar{q}_{3} \bar{q}_{4}\right)\right|^{2}\right\rangle=g^{4} \frac{4}{9}\left[\frac{s^{2}+u^{2}}{t^{2}}+\frac{s^{2}+t^{2}}{u^{2}}-\frac{2}{3} \frac{s^{2}}{t u}\right]|\widetilde{V}(t, u)|^{2}
\end{aligned}
$$

Each scattering matrix element squared (25) 27) contains two types of terms. The first type for two quarks of one chirality and two of the other chirality includes a sum over two independent helicity channels (not related by parity) of single helicity amplitudes squared in a single color channel. The second type for four quarks of the same chirality includes a sum over the same two independent helicity channels of the square of a coherent sum of two helicity amplitudes in different color channels.

\subsubsection{Four Gluons}

The unique four gluon color ordered helicity amplitudes may be written

$$
\begin{aligned}
& \mathcal{A}\left(g_{1}^{-a}, g_{2}^{+b}, g_{3}^{ \pm c}, g_{4}^{\mp d}\right)=A\left(g_{1}^{-}, g_{2}^{+}, g_{3}^{ \pm}, g_{4}^{\mp}\right) \operatorname{Tr}\left(T^{a} T^{b} T^{c} T^{d}\right) \\
& \mathcal{A}\left(g_{1}^{-a}, g_{2}^{-b}, g_{3}^{+c}, g_{4}^{+d}\right)=A\left(g_{1}^{-}, g_{2}^{-}, g_{3}^{+}, g_{4}^{+}\right) \operatorname{Tr}\left(T^{a} T^{b} T^{c} T^{d}\right)
\end{aligned}
$$

with others related by parity. The QCD helicity amplitudes with color factors stripped off are all related by permutations to the Parke-Taylor amplitude [24]

$$
\begin{aligned}
& A\left(g_{1}^{-}, g_{2}^{+}, g_{3}^{+}, g_{4}^{-}\right)_{\mathrm{QCD}}=g^{2} \frac{\langle 14\rangle^{4}}{\langle 12\rangle\langle 23\rangle\langle 34\rangle\langle 41\rangle} \\
& A\left(g_{1}^{-}, g_{2}^{+}, g_{3}^{-}, g_{4}^{+}\right)_{\mathrm{QCD}}=g^{2} \frac{\langle 13\rangle^{4}}{\langle 12\rangle\langle 23\rangle\langle 34\rangle\langle 41\rangle} \\
& A\left(g_{1}^{-}, g_{2}^{-}, g_{3}^{+}, g_{4}^{+}\right)_{\mathrm{QCD}}=g^{2} \frac{\langle 12\rangle^{4}}{\langle 12\rangle\langle 23\rangle\langle 34\rangle\langle 41\rangle}
\end{aligned}
$$

These color ordered helicity amplitudes may be averaged (summed) over initial (final) state color and helicity configurations to give the averaged matrix elements squared for $2 \rightarrow 2$ scattering processes involving four gluons with the form factor modification

$$
\begin{aligned}
\left\langle\left|\mathcal{A}\left(g_{1} g_{2} \rightarrow g_{3} g_{4}\right)\right|^{2}\right\rangle=g^{4} \frac{9}{4}\left(\frac{1}{s^{2}}+\frac{1}{t^{2}}+\frac{1}{u^{2}}\right) & {\left[s^{2}|\widetilde{V}(t, u)|^{2}+t^{2}|\widetilde{V}(s, u)|^{2}+u^{2}|\widetilde{V}(s, t)|^{2}\right.} \\
& \left.-\frac{4}{27}|s \tilde{V}(t, u)+t \widetilde{V}(s, u)+u \widetilde{V}(s, t)|^{2}\right]
\end{aligned}
$$

The scattering matrix element squared (33) includes a sum over three independent helicity channels (not related by parity) of the square of a coherent sum of three helicity amplitudes each in a different color channel. 


\subsubsection{Two Quarks and two Gluons}

The unique color ordered helicity amplitudes for two quarks of a given flavor and chirality and two gluons may be written

$$
\mathcal{A}\left(\bar{q}_{1}^{-i}, q_{2}^{+j}, g_{3}^{ \pm a}, g_{4}^{\mp b}\right)=A\left(\bar{q}_{1}^{-}, q_{2}^{+}, g_{3}^{ \pm}, g_{4}^{\mp}\right)\left(T^{a} T^{b}\right)_{j}{ }^{i}
$$

with others related by charge conjugation or parity. The QCD helicity amplitudes with color factors stripped off are all related by permutations to a single amplitude

$$
\begin{aligned}
& A\left(\bar{q}_{1}^{-}, q_{2}^{+}, g_{3}^{+}, g_{4}^{-}\right)_{\mathrm{QCD}}=g^{2} \frac{\langle 14\rangle^{3}\langle 24\rangle}{\langle 12\rangle\langle 23\rangle\langle 34\rangle\langle 41\rangle} \\
& A\left(\bar{q}_{1}^{-}, q_{2}^{+}, g_{3}^{-}, g_{4}^{+}\right)_{\mathrm{QCD}}=g^{2} \frac{\langle 13\rangle^{3}\langle 23\rangle}{\langle 12\rangle\langle 23\rangle\langle 34\rangle\langle 41\rangle}
\end{aligned}
$$

These color ordered helicity amplitudes may be averaged (summed) over initial (final) state color and helicity configurations to give the averaged matrix elements squared for $2 \rightarrow 2$ scattering processes involving two quarks of a given flavor and two gluons with the form factor modification

$$
\begin{aligned}
& \left\langle\left|\mathcal{A}\left(q_{1} \bar{q}_{2} \rightarrow g_{3} g_{4}\right)\right|^{2}\right\rangle=g^{4} \frac{32}{27} \frac{u^{2}+t^{2}}{s^{2}}\left[\frac{u}{t}|\widetilde{V}(s, t)|^{2}+\frac{t}{u}|\widetilde{V}(s, u)|^{2}-\frac{1}{4} \operatorname{Re}\left(\widetilde{V}(s, t) \widetilde{V}^{*}(s, u)\right)\right] \\
& \left\langle\left|\mathcal{A}\left(g_{1} g_{2} \rightarrow q_{3} \bar{q}_{4}\right)\right|^{2}\right\rangle=g^{4} \frac{1}{6} \frac{u^{2}+t^{2}}{s^{2}}\left[\frac{u}{t}|\widetilde{V}(s, t)|^{2}+\frac{t}{u}|\widetilde{V}(s, u)|^{2}-\frac{1}{4} \operatorname{Re}\left(\widetilde{V}(s, t) \widetilde{V}^{*}(s, u)\right)\right] \\
& \left\langle\left|\mathcal{A}\left(q_{1} g_{2} \rightarrow q_{3} g_{4}\right)\right|^{2}\right\rangle=g^{4} \frac{4}{9} \frac{s^{2}+u^{2}}{t^{2}}\left[-\frac{s}{u}|\widetilde{V}(t, u)|^{2}-\frac{u}{s}|\widetilde{V}(s, t)|^{2}+\frac{1}{4} \operatorname{Re}\left(\widetilde{V}(s, t) \widetilde{V}^{*}(t, u)\right)\right] \\
& \left\langle\left|\mathcal{A}\left(\bar{q}_{1} g_{2} \rightarrow \bar{q}_{3} g_{4}\right)\right|^{2}\right\rangle=g^{4} \frac{4}{9} \frac{s^{2}+u^{2}}{t^{2}}\left[-\frac{s}{u}|\widetilde{V}(t, u)|^{2}-\frac{u}{s}|\widetilde{V}(s, t)|^{2}+\frac{1}{4} \operatorname{Re}\left(\widetilde{V}(s, t) \widetilde{V}^{*}(t, u)\right)\right]
\end{aligned}
$$

Each scattering matrix element squared 37, 40 includes a sum over two independent helicity channels (not related by parity) of the square of a coherent sum of two helicity amplitudes in different color channels.

\subsection{Behavior of the Form Factor}

The behavior of the Veneziano form factor model defined in (14) and (15) depends on the damping parameter $\alpha$. For $\alpha<1$ the form factor includes Regge resonances representing the effects of intermediate perturbative string states, and does not satisfy the extinction 
property (4). For $\alpha=1$ the extinction property (4) is obtained, with the modulus of the Veneziano factors for central scattering in all kinematic channels monotonically decreasing as a function of the center of mass energy, whereas for $\alpha>1$ this feature is lost. So for definiteness we take a universal value of $\alpha=1$ to model extinction of all QCD $2 \rightarrow 2$ scattering processes. In this case the form factor with large absorptive branch cut effectively represents a continuum of strongly self coupled high entropy intermediate states. This is the regime that is relevant to use of the Veneziano form factor as a model of extinction.

The effects of the extinction Veneziano form factor modification of various QCD $2 \rightarrow 2$ scattering probabilities with $\alpha=1$ for central scattering corresponding to $t=u=-s / 2$ are shown in Fig. 2. The rapid decrease in effective overlap of the intermediate states with low multiplicity states is apparent in the extinction of central $2 \rightarrow 2$ scattering for center of mass energies beyond the Veneziano extinction mass scale $M_{E}$. The extinction of scattering processes with both a quark and anti-quark in the initial or final states is more rapid than that for the other processes because the former involve only $\widetilde{V}(s, t)$ or $\widetilde{V}(s, u)$, while the latter include also $\widetilde{V}(t, u)$ which falls more slowly for central scattering.

The dominant QCD $2 \rightarrow 2$ scattering process for proton-proton collisions at high energy is $q g \rightarrow q g$. The effects of the extinction Veneziano form factor modification on this process with $\alpha=1$ are shown in Fig. 3 as a function of center of mass energy and scattering angle. The extinction for scattering energies above the Veneziano mass scale $M_{E}$ is most pronounced for central scattering, with minimal extinction for scattering near the forward and backward directions. The magnitude of extinction correlates well with the transverse momentum of either of the scattered partons, as shown in Fig. 3. The transverse momentum is a rather direct measure of the energy or length scale probed in the scattering process. And extinction of hard scattering processes in proportion to the transverse momentum is expected on general grounds for scattering energies above the fundamental Planck scale, or equivalently scattering distances below the Planck length. The fairly tight correlation between extinction and transverse momentum is another nice feature of the Veneziano form factor model of extinction used here. And on general grounds, for scattering into final states of any multiplicity, the transverse momentum of the leading jet represents a useful kinematic variable in which to characterize the degree of extinction.

\section{Jet Extinction at the LHC}

Searching for jet extinction from non-perturbative quantum gravity effects at the LHC requires a quantitative extinction model to compare against data. In the next subsec- 


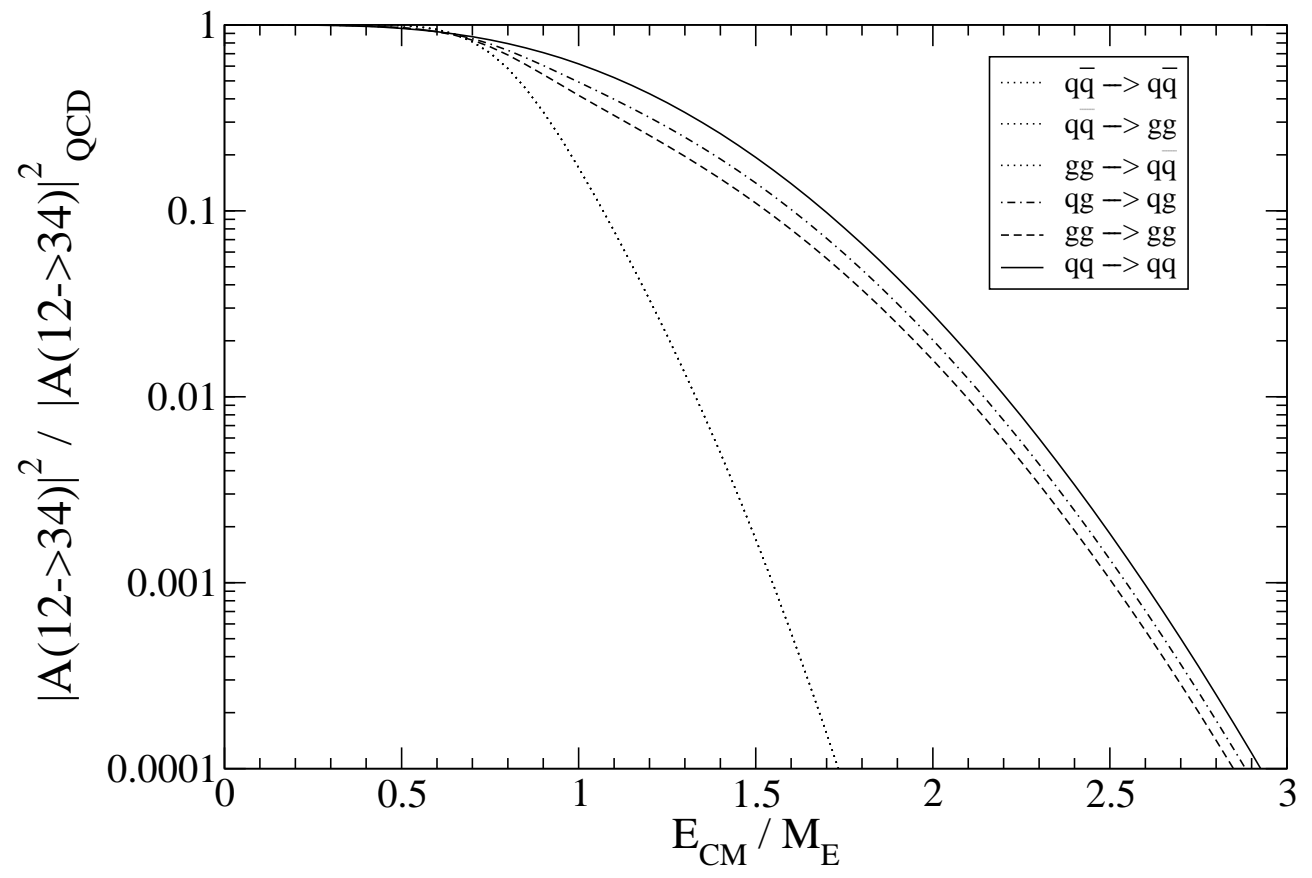

Figure 2: Effects of the Veneziano form factor extinction with $\alpha=1$ on various $2 \rightarrow 2$ quark and gluon scattering probabilities $|\mathcal{A}(12 \rightarrow 34)|^{2} /|\mathcal{A}(12 \rightarrow 34)|_{\mathrm{QCD}}^{2}$ for central scattering $u=t=-s / 2$ as a function of the center of mass energy $E_{\mathrm{CM}}=\sqrt{s}$ in units of the extinction mass scale $M_{E}$. 


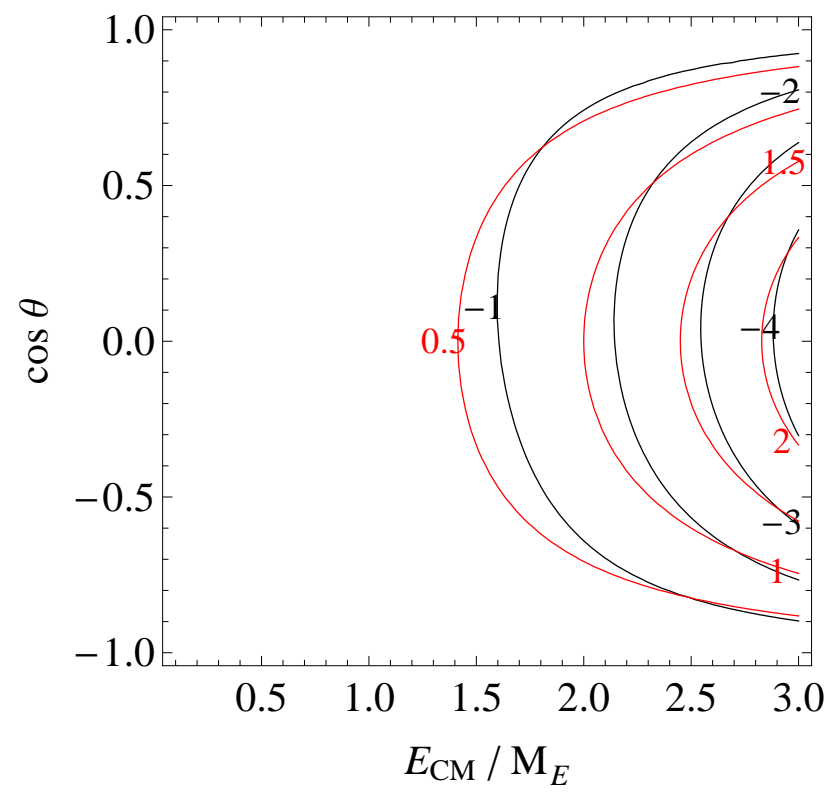

Figure 3: Effect of the Veneziano form factor extinction with $\alpha=1$ on the $q g \rightarrow q g$ scattering probability as a function of the center of mass mass energy, $E_{\mathrm{CM}}=\sqrt{s}$, and scattering angle, $\cos \theta=1+2 t / s$. Black contours indicate $|\mathcal{A}(q g \rightarrow q g)|^{2} /|\mathcal{A}(q g \rightarrow q g)|_{\mathrm{QCD}}^{2}$ labelled in $\log _{10}$ units. Red contours indicate $p_{T}^{2} / M_{E}^{2}=(s / 4)\left(1-\cos ^{2} \theta\right) / M_{E}^{2}$ in linear units. 
tion we describe an LO Extinction Monte Carlo (EMC) modification of the Pythia Monte Carlo event generator that implements the QCD $2 \rightarrow 2$ scattering matrix elements with Veneziano form factors presented in section 2. In the following subsection we use the EMC to estimate the statistical sensitivity of a search for extinction in the inclusive jet transverse momentum spectrum. We also describe how the results of the EMC could be conservatively implemented as a phenomenological extinction form factor modification of NLO QCD predictions for the inclusive jet transverse momentum spectrum.

\subsection{Monte Carlo Implementation}

In order to study quantitative effects of extinction at the LHC with an EMC event generator, we incorporated Veneziano form factors for QCD scattering processes into Pythia 6.4 [25]. The standard QCD $2 \rightarrow 2$ scattering processes in Pythia appear in subroutine PYSGQC in the form

$$
\frac{d \sigma}{d \hat{t}}(12 \rightarrow 34)=\frac{1}{16 \pi \hat{s}^{2}}\left\langle|\mathcal{A}(12 \rightarrow 34)|^{2}\right\rangle \frac{1}{S}
$$

where $S=1(1 / 2)$ is a symmetry factor for distinct(identical) particles in the final state, and $\hat{t}$ is integrated over the region $-\hat{s} \leq \hat{t} \leq 0$. We modified the squared matrix elements in PYSGQC for each QCD $2 \rightarrow 2$ scattering process to include the Veneziano form factors given in section 2.1. The gamma functions with complex arguments in the Veneziano amplitude were evaluated numerically in a separate subroutine. With this modification we are able to generate events corresponding to a given extinction scenario with Veneziano form factor scale $M_{E}$ and damping parameter $\alpha$, and interface the events to the (unmodified) parton shower and hadronization subroutines of Pythia. The leading initial and final state QCD radiation effects in this extinction model are therefore included in the Pythia event generation. Since the energy scale for most radiation processes are well below the hard scattering scale (which because of the rapid extinction is effectively at most of order the extinction scale) this treatment of radiation effects should be a good approximation, and amounts formally to including next to leading logarithmic (NLL) effects without extinction. This realization of an EMC has the advantage that in the limit of large extinction scale $M_{E}$, the results reduce to those of Pythia 6.4, which has been well tested and validated.

To model detector effects, such as acceptance and jet energy resolution, the hadronized events are passed through the PGS detector simulation [26]. We use the default CMS parameter set of PGS, with a jet definition based on a cone algorithm with cone size $R=0.7$. 


\subsection{Searching for Jet Extinction}

The general infrared-ultraviolet properties of non-perturbative quantum gravity imply that hard scattering processes should be suppressed for scattering energies above the fundamental Planck scale. Any observable used in a search for extinction at the LHC must be measured as a function of some kinematic invariant that characterizes the hard energy scale in the scattering process. The simplest and most inclusive kinematic variable that characterizes the energy scale in QCD scattering at a hadron collider is jet transverse momentum. The simplest inclusive observable as a function of this energy scale is the overall rate. And as discussed above, the extinction effects in the Veneziano form factor model with large absorptive branch cut are well correlated with transverse momentum. We therefore propose that a search for extinction could be accomplished by a careful comparison of a high integrated luminosity measurement of the inclusive jet transverse momentum spectrum with QCD expectations. This spectrum is a robust inclusive observable that can be calculated rather reliably in QCD at NLO [27, 28, 29] and has already been measured at the LHC by the CMS and ATLAS collaborations with relatively low integrated luminosity [30, 31]. An extinction search of this type could benefit from experience gained in these previous measurements, and could be carried out as an interpretation of future high luminosity measurements of the inclusive jet transverse momentum spectrum.

In order to illustrate the leading effects of extinction at the LHC on the inclusive jet transverse momentum spectrum we use the EMC modification of the Pythia event generator described in the previous subsection. For event selection we require at least two jets, each with transverse momentum $p_{T}>500 \mathrm{GeV}$ and pseudo-rapidity $|\eta|<1.5$. This yields hard scattering events with relatively large center of mass scattering angle. We simulate protonproton collisions for both $7 \mathrm{TeV}$ and $13 \mathrm{TeV}$ center of mass energies. For $7 \mathrm{TeV}$ we generate QCD events without any extinction in $\sqrt{\hat{s}}$ bins of $300 \mathrm{GeV}$, starting at $\sqrt{\hat{s}}=200 \mathrm{GeV}$, each bin containing 50000 events. We use the same $\sqrt{\hat{s}}$ bins to simulate extinction with 25000 events in each bin for Veneziano mass scales of $M_{E}=1,1.5,2$, and $3 \mathrm{TeV}$ with $\alpha=1$. For $13 \mathrm{TeV}$ we use $\sqrt{\hat{s}}$ bins of $500 \mathrm{GeV}$ starting at $\sqrt{\hat{s}}=500 \mathrm{GeV}$, with 100000 events in each bin for QCD, and 25000 events per bin for Veneziano mass scales of $M_{E}=2,3,4$, and $5 \mathrm{TeV}$ with $\alpha=1$. The differential cross section spectra as a function of the leading jet transverse momentum for these cases are shown in Figs. 4 and 5 . The extinction

spectra drop to half of the QCD expectation for a transverse mass of roughly one half of the extinction mass scale.

The statistical significance of the extinction of the inclusive jet transverse momentum 


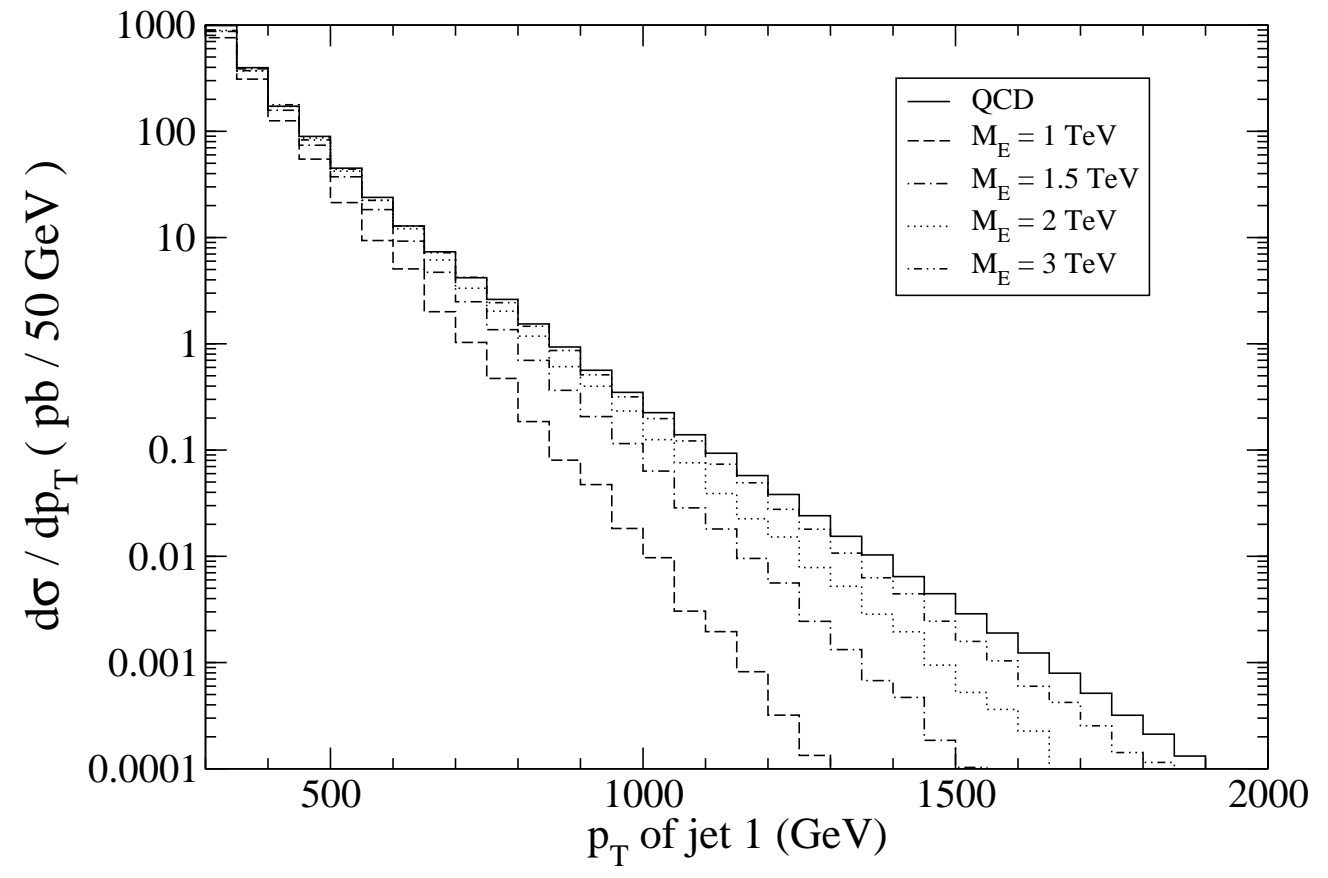

Figure 4: The differential cross section as a function of the transverse momentum of the leading jet for various values of the Veneziano extinction mass scale $M_{E}$ with $\alpha=1$ for 7 $\mathrm{TeV}$ proton-proton collisions after the selection cuts described in the text. 


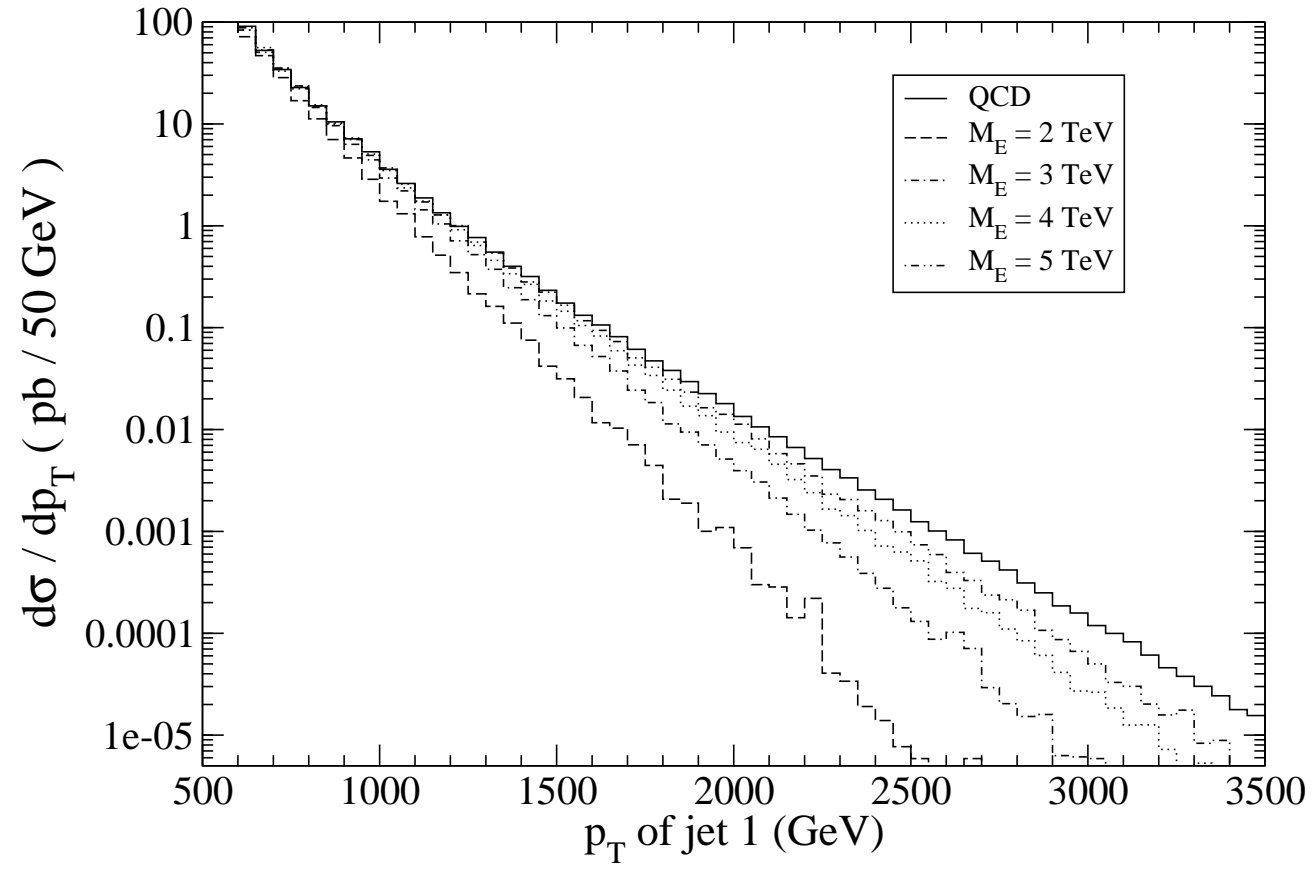

Figure 5: The differential cross section as a function of the transverse momentum of the leading jet for various values of the Veneziano extinction mass scale $M_{E}$ with $\alpha=1$ for 13 $\mathrm{TeV}$ proton-proton collisions after the selection cuts described in the text. 
spectrum as compared with QCD expectations for a given Veneziano extinction mass scale, center of mass collision energy, and integrated luminosity can be assessed with a simple likelihood analysis. To do this we divide the leading jet transverse momenta into $100 \mathrm{GeV}$ bins above $500 \mathrm{GeV}$ for $7 \mathrm{TeV}$ collisions, and above $1500 \mathrm{GeV}$ for $13 \mathrm{TeV}$ collisions. Using the calculated values of the differential cross sections we define probability distributions across the bins for QCD and the extinction hypotheses labeled by the Veneziano mass scale $M_{E}$

$$
P\left(i \mid M_{E}\right)=\frac{d \sigma\left(i \mid M_{E}\right) / d p_{T}}{\sum_{i} d \sigma\left(i \mid M_{E}\right) / d p_{T}}
$$

where $i$ labels the $100 \mathrm{GeV}$ wide $p_{T}$ bin, and where QCD corresponds to $M_{E}=\infty$. Note that the overall normalization drops out, so the probability distributions $P\left(i \mid M_{E}\right)$ are sensitive to shape only. A joint likelihood entropy functional between a probability distribution for a given extinction scale $M_{E}$ and a data set corresponding to an extinction scale $M_{E}^{\prime}$ obtained with an integrated luminosity $L$ may be defined as being proportional to the logarithm of the total joint probability

$\mathcal{S}\left[P\left(i \mid M_{E}\right), N\left(i \mid M_{E}^{\prime}, L\right)\right]=-2 \ln \left(\prod_{i} P\left(i \mid M_{E}\right)^{N\left(i \mid M_{E}^{\prime}, L\right)}\right)=-2 \sum_{i} N\left(i \mid M_{E}^{\prime}, L\right) \ln P\left(i \mid M_{E}\right)$

where $N\left(i \mid M_{E}^{\prime}, L\right)$ is the number of events in the $i$-th $p_{T}$ bin. For any data set corresponding to an extinction scale $M_{E}^{\prime}$, the difference in likelihood entropy functionals for probability distributions corresponding to extinction scale $M_{E}$ and QCD, or equivalently the logarithmic likelihood ratio of extinction to QCD,

$Q\left[P\left(i \mid M_{E}\right), P(i \mid \mathrm{QCD}), N\left(i \mid M_{E}^{\prime}, L\right)\right]=\mathcal{S}\left[P\left(i \mid M_{E}\right), N\left(i \mid M_{E}^{\prime}, L\right)\right]-\mathcal{S}\left[P(i \mid \mathrm{QCD}), N\left(i \mid M_{E}^{\prime}, L\right)\right]$

may be used to quantify the statistical separation between an extinction hypothesis and QCD.

For each extinction mass scale presented in Figs. 4 and 5 and for QCD we generated normalized statistical probability distributions

$$
\mathcal{P}\left(Q \mid M_{E}, L\right) \equiv \frac{d P\left(Q \mid M_{E}, L\right)}{d Q}
$$

of the joint likelihood entropy differences

$$
Q\left[N\left(i \mid M_{E}, L\right)\right] \equiv Q\left[P\left(i \mid M_{E}\right), P(i \mid \mathrm{QCD}), N\left(i \mid M_{E}, L\right)\right]
$$

based on data from 50000 pseudo-experiments, where the number of events $N\left(i \mid M_{E}, L\right)$ in each $p_{T}$ bin of width $\Delta p_{T}$ is chosen randomly from a Poisson distribution with average 
expected events $\left\langle N\left(i \mid M_{E}, L\right)\right\rangle=L \cdot \Delta p_{T} \cdot d \sigma\left(i \mid M_{E}\right) / d p_{T}$. For $7 \mathrm{TeV}$ collisions we take an integrated luminosity of $L=5 \mathrm{fb}^{-1}$ and for $13 \mathrm{TeV}$ collisions $L=100 \mathrm{fb}^{-1}$. Using a Gaussian fit, $\widetilde{\mathcal{P}}\left(Q \mid M_{E}, L\right)$, to each distribution we determine the value of the likelihood entropy difference $Q_{0}\left(M_{E}, L\right)$ where the extinction and QCD data set distributions have the same statistical probability for a given integrated luminosity

$$
\widetilde{\mathcal{P}}\left(Q_{0} \mid M_{E}, L\right)=\widetilde{\mathcal{P}}\left(Q_{0} \mid \mathrm{QCD}, L\right)
$$

Data sets with values of $Q\left[N\left(i \mid M_{E}, L\right)\right]>Q_{0}\left(M_{E}, L\right)$ are more likely to be consistent with QCD, and ones with $Q\left[N\left(i \mid M_{E}, L\right)\right]<Q_{0}\left(M_{E}, L\right)$ are more likely to be consistent with extinction. The statistical probability that a QCD data set is more consistent with an extinction hypothesis with extinction scale $M_{E}$ than with QCD is then

$$
P\left(\mathrm{QCD} \mid M_{E}, L\right)=\int_{-\infty}^{Q_{0}} d Q \widetilde{\mathcal{P}}(Q \mid \mathrm{QCD}, L)
$$

and that an extinction data set with extinction scale $M_{E}$ is more consistent with the QCD hypothesis than extinction is

$$
P\left(M_{E} \mid \mathrm{QCD}, L\right)=\int_{Q_{0}}^{+\infty} d Q \widetilde{\mathcal{P}}\left(Q \mid M_{E}, L\right)
$$

These two mis-identification probabilities need not be equal, and to be conservative we consider the larger of the two. For $7 \mathrm{TeV}$ collisions with $5 \mathrm{fb}^{-1}$ of integrated luminosity we find that the statistical mis-identification probability is below $5.7 \times 10^{-7}$ corresponding to $5 \sigma$ significance for an extinction scale of $M_{E} \leq 3 \mathrm{TeV}$. And for $13 \mathrm{TeV}$ collisions with 100 $\mathrm{fb}^{-1}$ of integrated luminosity we find similar statistical significance for $M_{E} \leq 5 \mathrm{TeV}$. These rather conservative estimates include statistical uncertainties only. An experimental search would also include detector systematic effects such as from jet energy scale and resolution uncertainties that are beyond the scope of this illustrative study. But based on the results presented here, it seems reasonable to expect that high luminosity searches could probe extinction scales up to roughly half the center of mass beam collision energy.

The Veneziano form factor model presented in section 2 and utilized above includes extinction effects only for QCD $2 \rightarrow 2$ hard scattering processes. Leading initial and final state QCD radiation effects in this model are included formally at NLL approximation in the EMC modification of Pythia discussed in section 3.1. Full NLO effects for extinction of hard $2 \rightarrow 3$ and higher multiplicity scattering processes are, however, not included. Experimental measurements of the inclusive jet transverse momentum spectrum [30, 31] 
do make comparisons with available NLO QCD calculations [27, 28, 29]. So it would be useful to present the effects of extinction as a modification of these NLO predictions of the form

$$
\frac{d \sigma}{d p_{T}}\left(p p \rightarrow \text { jets } \mid p_{T}, M_{E}\right) \simeq F\left(p_{T}, M_{E}\right) \frac{d \sigma}{d p_{T}}\left(p p \rightarrow \text { jets } \mid p_{T}\right)_{\mathrm{NLO}}
$$

where $F\left(p_{T}, M_{E}\right)$ is a phenomenological form factor function that depends on the extinction scale $M_{E}$. As a practical matter the magnitude of the extinction scale that can be probed in proton-proton collisions is limited roughly by the highest transverse momenta jets where the data begins to run out. In this regime where scattering energies are by definition at most of order the extinction scale, the leading effects of extinction on energetic grounds are for the leading $2 \rightarrow 2$ hard scattering processes. Higher multiplicity processes at the same transverse momentum probe lower individual scattering energies with smaller extinction effects. So a conservative ansatz that would be useful in quantifying the onset of extinction would be to assume that extinction is limited to $2 \rightarrow 2$ hard scattering processes. Inclusion of extinction effects for higher multiplicity hard scattering processes would only soften the transverse momentum spectrum. For a given data set that is roughly consistent with NLO QCD expectations, inclusion of these sub-dominant effects would only yield a stronger bound. This ansatz amounts to an $F\left(p_{T}, M_{E}\right)$ of the form

$$
F\left(p_{T}, M_{E}\right)=\left[1-C_{\mathrm{NLO}}\left(p_{T}\right)\right] f_{\mathrm{LO}}\left(p_{T}, M_{E}\right)+C_{\mathrm{NLO}}\left(p_{T}\right)
$$

where $f_{\mathrm{LO}}\left(p_{T}, M_{E}\right)$ represents extinction of LO $2 \rightarrow 2$ scattering procesess, and the $p_{T}$ dependent NLO fraction for $p p \rightarrow$ jets is

$$
C_{\mathrm{NLO}}\left(p_{T}\right)=1-\frac{\left(d \sigma / d p_{T}\right)_{\mathrm{LO}}}{\left(d \sigma / d p_{T}\right)_{\mathrm{NLO}}}
$$

NLO QCD calculations of the inclusive jet transverse momentum spectrum [27, 28, 29] generally include a numerical comparison of LO and NLO results that may be used to extract $C_{\mathrm{NLO}}\left(p_{T}\right)$. A functional form for the leading order extinction function $f_{\mathrm{LO}}\left(p_{T}, M_{E}\right)$ may be obtained by comparing the results of the EMC modification of Pythia described in section 3.1 for different $M_{E}$ with the Pythia QCD results for $M_{E}=\infty$. The modulus squared of the Veneziano form factor for central scattering in all kinematic channels has the general form of a sigmoid function. We have found that a simple sigmoid gives a good functional fit to the $p_{T} \lesssim \mathcal{O}\left(M_{E}\right)$ onset of LO extinction in the Veneziano model

$$
f_{\mathrm{LO}}\left(p_{T}, M_{E}\right)=\frac{1}{1+\exp \left[\left(p_{T}-p_{T, 1 / 2}\right) / p_{T, 0}\right]}
$$


where $p_{T, 1 / 2}=f_{1 / 2}\left(M_{E}\right)$ and $p_{T, 0}=f_{0}\left(M_{E}\right)$ are $M_{E}$ dependent functions that must be determined by fitting to Monte Carlo results. The function $f_{1 / 2}\left(M_{E}\right)$ is the value of $p_{T}$ for a given $M_{E}$ where the LO inclusive jet spectrum drops to half of the QCD expectation, and should be linear in $M_{E}$. The function $f_{0}\left(M_{E}\right)$ is the rate of change of the extinction with $p_{T}$, and should be well fit by constant plus linear term in $M_{E}$. This phenomenological form factor modification of NLO QCD predictions should be sufficient to quantify the results of future searches for the onset of extinction in the inclusive jet transverse momentum spectrum.

\section{Conclusions}

The infrared-ultraviolet holographic properties of quantum gravity suggest on very general grounds that at scattering energies beyond the fundamental Planck scale, low multiplicity final states suffer rapid extinction. If the fundamental Planck scale is not too far above the electroweak scale, this interesting feature of non-perturbative quantum gravity could in principle be subject to experimental investigation in the laboratory. In this paper we proposed that these extinction effects could be searched for in the inclusive jet transverse momentum spectrum at the LHC. We presented a large damping Veneziano form factor extinction model of QCD $2 \rightarrow 2$ scattering processes, and implemented this into a modified version of the Pythia event generator to simulate extinction processes. This model has the nice feature that it smoothly reduces to Pythia QCD scattering as the Veneziano extinction scale is taken large. Based on simulations using these tools, we estimate that future high luminosity measurements of the inclusive jet transverse momentum spectrum could be statistically sensitive to an extinction scale of order half of the beam center of mass collision energy. We also suggested a simple and conservative phenomenological form factor modification of NLO QCD predictions for this spectrum that could be used to quantify future searches for the onset of extinction.

The search for jet extinction from non-perturbative quantum gravity effects in high energy collisions at the LHC is complementary to ongoing searches for the related phenomenon of excess production of high multiplicity final states. Since the intrinsic sensitivity of these searches depends on details of the underlying theory of quantum gravity, both should be part of the search program for strong gravitational effects at the LHC. Observation of either, or both, would open up a fantastic new experimental window into the holographic properties of gravity. 


\section{Acknowledgments}

We would like to thank J.P. Chou for useful comments. This research was supported in part by DOE grant DE-FG02-96ER40959 and NSF Grant PHY-0969020.

\section{References}

[1] T. Banks and W. Fischler, "A Model for High-Energy Scattering in Quantum Gravity," hep-th/9906038.

[2] For a recent review of scattering in theories of quantum gravity see S. B. Giddings, "The gravitational S-matrix: Erice lectures," arXiv:1105.2036 [hep-th].

[3] S. B. Giddings and S. D. Thomas, "High-Energy Colliders as Black Hole Factories: The End of Short Distance Physics," Phys. Rev. D 65, 056010 (2002) hep-ph/0106219.

[4] N. Arkani-Hamed, S. Dimopoulos and G. R. Dvali, "The Hierarchy problem and new dimensions at a millimeter," Phys. Lett. B 429, 263 (1998) hep-ph/9803315].

[5] I. Antoniadis, N. Arkani-Hamed, S. Dimopoulos and G. R. Dvali, "New dimensions at a millimeter to a Fermi and superstrings at a TeV," Phys. Lett. B 436, 257 (1998) hep-ph/9804398.

[6] L. Randall and R. Sundrum, "A Large mass hierarchy from a small extra dimension," Phys. Rev. Lett. 83, 3370 (1999) [hep-ph/9905221].

[7] S. Dimopoulos and G. L. Landsberg, "Black holes at the LHC," Phys. Rev. Lett. 87, 161602 (2001) hep-ph/0106295].

[8] P. Meade and L. Randall, "Black Holes and Quantum Gravity at the LHC," JHEP 0805, 003 (2008) arXiv:0708.3017 [hep-ph]].

[9] V. Khachatryan et al. [CMS Collaboration], "Search for Microscopic Black Hole Signatures at the Large Hadron Collider," Phys. Lett. B 697, 434 (2011) arXiv:1012.3375 [hep-ex]].

[10] S. Chatrchyan et al. [CMS Collaboration], "Search for microscopic black holes in $p p$ collisions at $\sqrt{s}=7 \mathrm{TeV}$," JHEP 1204, 061 (2012) arXiv:1202.6396 [hep-ex]]. 
[11] G. Aad et al. [ATLAS Collaboration], "Search for strong gravity signatures in samesign dimuon final states using the ATLAS detector at the LHC," Phys. Lett. B 709, 322 (2012) arXiv:1111.0080 [hep-ex]].

[12] G. Aad et al. [ATLAS Collaboration], "Search for TeV-scale gravity signatures in final states with leptons and jets with the ATLAS detector at $\sqrt{s}=7 \mathrm{TeV}$," Phys. Lett. B 716, 122 (2012) arXiv:1204.4646 [hep-ex]].

[13] G. F. Giudice, R. Rattazzi and J. D. Wells, "Quantum gravity and extra dimensions at high-energy colliders," Nucl. Phys. B 544, 3 (1999) hep-ph/9811291.

[14] E. A. Mirabelli, M. Perelstein and M. E. Peskin, "Collider signatures of new large space dimensions," Phys. Rev. Lett. 82, 2236 (1999) hep-ph/9811337.

[15] S. Cullen, M. Perelstein and M. E. Peskin, "TeV strings and collider probes of large extra dimensions," Phys. Rev. D 62, 055012 (2000) hep-ph/0001166.

[16] D. Lust, S. Stieberger and T. R. Taylor, "The LHC String Hunter's Companion," Nucl. Phys. B 808, 1 (2009) arXiv:0807.3333 [hep-th]].

[17] V. Khachatryan et al. [CMS Collaboration], "Search for Dijet Resonances in $7 \mathrm{TeV} p p$ Collisions at CMS," Phys. Rev. Lett. 105, 211801 (2010) arXiv:1010.0203 [hep-ex]].

[18] S. Chatrchyan et al. [CMS Collaboration], "Search for Resonances in the Dijet Mass Spectrum from $7 \mathrm{TeV}$ pp Collisions at CMS," Phys. Lett. B 704, 123 (2011) arXiv:1107.4771 [hep-ex]].

[19] G. Aad et al. [ATLAS Collaboration], "ATLAS search for new phenomena in dijet mass and angular distributions using $p p$ collisions at $\sqrt{s}=7 \mathrm{TeV}$," JHEP 1301, 029 (2013) arXiv:1210.1718 [hep-ex]].

[20] S. Chatrchyan et al. [CMS Collaboration], "Search for narrow resonances and quantum black holes in inclusive and $b$-tagged dijet mass spectra from $p p$ collisions at $\sqrt{s}=7$ TeV," JHEP 1301, 013 (2013) arXiv:1210.2387 [hep-ex]].

[21] G. Veneziano, "Construction of a crossing - symmetric, Regge behaved amplitude for linearly rising trajectories," Nuovo Cim. A 57, 190 (1968).

[22] D. J. Gross and P. F. Mende, "The High-Energy Behavior of String Scattering Amplitudes," Phys. Lett. B 197, 129 (1987). 
[23] D. J. Gross and P. F. Mende, "String Theory Beyond the Planck Scale," Nucl. Phys. B 303, 407 (1988).

[24] S. J. Parke and T. R. Taylor, "An Amplitude for $n$ Gluon Scattering," Phys. Rev. Lett. 56, 2459 (1986).

[25] T. Sjostrand, S. Mrenna, P. Z. Skands, "PYTHIA 6.4 Physics and Manual," JHEP 0605, 026 (2006). hep-ph/0603175].

[26] J. Conway et al., "PGS 4: Pretty Good Simulation of high energy collisions," 2006, www.physics. ucdavis. edu/ conway/research/software/pgs/pgs4-general.htm

[27] Z. Nagy, "Three jet cross-sections in hadron hadron collisions at next-to-leading order," Phys. Rev. Lett. 88, 122003 (2002) hep-ph/0110315].

[28] Z. Nagy, "Next-to-leading order calculation of three jet observables in hadron hadron collision," Phys. Rev. D 68, 094002 (2003) [hep-ph/0307268].

[29] T. Kluge, K. Rabbertz and M. Wobisch, "FastNLO: Fast pQCD calculations for PDF fits," hep-ph/0609285.

[30] S. Chatrchyan et al. [CMS Collaboration], "Measurement of the Inclusive Jet Cross Section in $p p$ Collisions at $\sqrt{s}=7 \mathrm{TeV}$," Phys. Rev. Lett. 107, 132001 (2011) arXiv:1106.0208 [hep-ex]].

[31] G. Aad et al. [ATLAS Collaboration], "Measurement of inclusive jet and dijet production in $p p$ collisions at $\sqrt{s}=7 \mathrm{TeV}$ using the ATLAS detector," Phys. Rev. D 86, 014022 (2012) arXiv:1112.6297 [hep-ex]]. 